\title{
Ángel Flores, agente doble del cosmopolitismo transatlántico
}

\author{
Ángel Flores, a Double Agent \\ for Transatlantic Cosmopolitanism
}

\author{
Juan Herrero-Senés \\ University of Colorado Boulder \\ herreros@colorado.edu \\ ORCID iD: https://orcid.org/0000-0002-5439-6472
}

\section{RESUMEN}

Este ensayo estudia la primera fase de la trayectoria del traductor y crítico literario puertorriqueño Ángel Flores, desde sus inicios hasta la Segunda Guerra Mundial. En esos años, Flores lleva a cabo una intensa campaña de difusión en Estados Unidos de los nuevos valores de la literatura europea, y particularmente española, a través de traducciones, antologías, publicaciones periódicas y contactos con escritores españoles. A la vez, Flores se convierte en una pieza importante en la expansión de la literatura norteamericana en España gracias sobre todo a su traducción de The Waste Land de T.S. Eliot. Se señalan sus principales aportaciones y se estudia de qué manera las preferencias estéticas del crítico fueron modificándose dando un giro hacia la literatura comprometida que permite explicar su interés posterior por las letras latinoamericanas.

Palabras Clave: Ángel Flores; T. S. Eliot; joven literatura española; Federico García Lorca; cosmopolitismo; hispanismo en Estados Unidos.

\begin{abstract}
This paper studies the first phase of the trajectory of the Puerto Rican translator and literary critic Ángel Flores, from the start up to the Second World War. In those years, Flores conducts an intensive publicity campaign in the United States of the new values of European literature, and particularly Spanish, through translations, anthologies, periodicals and personal contacts with Spanish writers. At the same time, Flores becomes an important part in the expansion of American literature in Spain thanks mainly to his translation of The Waste Land by T. S. Eliot. His main contributions are identified and studied to show how a change in his aesthetic preferences towards a literature of engagement explains his later interest in Latin American authors.
\end{abstract}

Key words: Ángel Flores; T. S. Eliot; Young Spanish Literature; Federico García Lorca; Cosmopolitanism; Hispanism in the US. 
El nombre de Ángel Flores (Barceloneta, Puerto Rico, 1900 - Guadalajara, México, 1992) se asocia en la historia literaria con varias referencias: suya es la primera traducción al español de The Waste Land de T. S. Eliot; está considerado el introductor de Franz Kafka en Estados Unidos; a él se debe la adaptación del concepto germano de «realismo mágico» a la literatura; se le tiene por uno de los artífices de la constitución de los estudios latinoamericanos como disciplina académica; y de su puño salieron incontables traducciones, ediciones, antologías y estudios de autores hispánicos, desde la poesía medieval hasta Octavio Paz y José Emilio Pacheco. Precisamente este último en un artículo de homenaje a Flores apuntó la característica central de su trayectoria que aquí pretendo analizar. Además de considerarlo «el más grande traductor bilingüe de nuestro siglo en el ámbito castellano» (1992: 48), Pacheco afirma que ejerce de «puente de circulación doble» (1992: 49)1 ${ }^{1}$. Y es que en sus roles de editor, traductor, crítico y profesor, Ángel Flores ocupó una posición pivotal en el campo literario transatlántico que hasta ahora nunca ha recibido atención crítica $^{2}$, construyendo una red de relaciones e intercambio entre los continentes de Europa y América. El presente artículo pretende ser un primer acercamiento al tema que ante la vastedad del panorama se circunscribe a la trayectoria de Flores hasta la Segunda Guerra Mundial. Constituyen esos años la época de exploración y primer afianzamiento de su posición en el campo literario y aquellos en los que, defiendo aquí, se forja el espíritu cosmopolita, cooperativo y dialógico que caracteriza su producción. Creo que precisamente el sentirse inmerso en un momento álgido de sincronía y colaboración transnacional y el querer ser parte activa en ella, además de una firme apuesta por los valores de la producción contemporánea, es lo que impulsó a Flores en su práctica crítica. Es en ese sentido que pertenecería a la categoría de «críticos cosmopolitas» en otro lugar (Herrero-Senés 2015) definidos como (aún escasamente reconocidos) agentes centrales del Modernismo (Modernism), concepto que de una vez debemos adoptar para hablar en términos generales de la producción cultural entre el fin del siglo XIX y la Segunda Guerra Mundial. La metáfora del «agente» referida a estos autores aúna tres ideas: indicar la importancia de su agencia, esto es, su rol de productores del diálogo crítico sobre las obras modernistas; su situación en segundo plano - como un agente secreto- en relación a los escritores de creación; y su labor como constructores de conexiones entre productores y editores, a la manera de un agente literario. Dotados de

${ }^{1}$ Metáfora que también se utiliza en la silueta de Ángel Flores sin firma que abre su libro póstumo Spanish American Authors: The 20th Century. («About Ángel Flores», Wilson, 1992, pp. xv-xvi).

2 Sobre Flores solo existen breves semblanzas como la de Josefina Cornejo (2012), el homenaje de José Emilio Pacheco (1992) y entrevistas como la de Miguel Ángel Flores (1991) y Laura Talavera (1992), junto a la entrevista sin firmar «En busca de Kafka», Nexos. 1-VI-1983, [s.p.], disponible en: http://www.nexos.com.mx/?p=4198 [ref. de 25/03/2016]. 
amplios conocimientos de lenguas (Flores dominaba una decena) y pasión por la producción dentro y fuera de su país, estos críticos a menudo dejaban de lado la escritura creativa o de ficción para concentrarse en tareas de diseminación, promoción y difusión de la literatura del momento. Apartaban como estorbos las divisiones nacionales, convencidos de la existencia de un espíritu común presente en las realizaciones culturales de su época, y se enorgullecían de ejercer de enlaces y de mediadores. En ese sentido tejían los hilos de las redes de intercambio intelectual forjadas, principalmente, entre Europa y América. Produjeron traducciones, antologías y recopilaciones, presentaron autores y movimientos, propusieron y moldearon lecturas, escribieron panoramas, introducciones, prólogos y reseñas, dieron noticias de los desarrollos de las distintas literaturas nacionales, ejercieron de representantes, asesores y evaluadores literarios, impulsaron publicaciones periódicas, dieron charlas, conferencias y cursos, se cartearon infatigablemente con autores, críticos y editores, intercambiaron materiales (libros, revistas, recortes, catálogos o manuscritos), señalaron tendencias, teorías y modas ordenando un abigarrado territorio, y se encontraron muy cómodos navegando entre los mares de papel de una producción literaria global plasmada en revistas grandes y pequeñas, panfletos y periódicos mayoritarios, tiradas limitadas e imperios editoriales, plataformas cuyo funcionamiento conocían a la perfección. Sabían que no existe literatura sin la materialidad y el estatus de mercancía y de intercambio de sus productos y realizaciones, y que es a través de estos canales y de una comunicación global que se constituyen, discuten y diseminan las ideas, los estilos, las preferencias y los prestigios, los éxitos y los fracasos, las leyes del mercado y las normas de la producción minoritaria. En ese sentido, los críticos cosmopolitas constituyen figuras centrales en la configuración y mapeo del espacio donde surge y se difunde la producción modernista.

El acercamiento a la labor de Flores, además de adentrarnos en este lado menos tratado del quehacer literario, nos plantea cuestiones de sociología literaria acerca de la canonización y promoción de ciertos autores en ciertos espacios, de cómo la literatura se disemina geográficamente, y alude, aun tangencialmente, a ese reverso de la historia literaria constituido no por sus realizaciones efectivas, sino por las ideaciones, ilusiones y proyectos frustrados, dicho en una línea, por los libros que nunca fueron. El recorrido que ahora empieza ofrece varios ejemplos de esta sombra de la vida literaria, y en el camino me apoyaré en varios momentos en el intercambio epistolar que Flores mantuvo con otro gigante de la crítica cosmopolita, Guillermo de Torre ${ }^{3}$. Lo que ambos se dicen

${ }^{3}$ El archivo personal de Guillermo de Torre conservado en la Biblioteca Nacional de España (MSS/22818-MSS/22843) incluye su correspondencia con Ángel Flores (MSS 22823), que comienza el 4 de octubre de 1929 hasta el año 1961, y a la que hago en este trabajo varias referencias. Agradezco a Domingo Ródenas de Moya la amabilidad de haberme proporcionado copias de las cartas. 
en sus cartas nos aporta datos centrales para observar la construcción de una red intelectual, y más concretamente para entender los propósitos e intereses de ambos críticos. En el caso de Flores (y de una forma distinta también para de Torre) estos intereses en los años del Modernismo se concentraron en construir y fortalecer la circulación de la producción intelectual entre Estados Unidos y Europa, y especialmente España. No fue hasta la década de los cuarenta que la actividad de Flores empezó a orientarse de una manera significativa en una dirección norte-sur, esto es, desde Estados Unidos hacia Latinoamérica. Como señaló acertadamente Guillermo de Torre en 1948, Flores tuvo una «primera vocación» dedicada a la literatura peninsular para luego acercarse más a Latinoamérica, a la par que recorría «el camino inverso» (1948: 18), dando a conocer en español obras significativas de la literatura norteamericana moderna.

Las dos fases de la trayectoria de Flores ponen además sobre el tapete la cuestión de la identidad y el sentimiento de pertenencia. El crítico puertorriqueño comienza a escribir en un complejo período donde los intelectuales latinoamericanos están solidificando su identidad colectiva a la vez que reformulando sus relaciones tanto con Europa como con Estados Unidos. Como es sabido, la eurofilia de tantos intelectuales latinoamericanos fue en los años de entreguerras dando paso a un progresivo acercamiento/fricción con Estados Unidos, y a un reforzamiento de las nociones de «panamericanismo» y de ideales de solidaridad continental, colaboración y cooperación intelectual entre las distintas naciones latinoamericanas. Como veremos, en sus primeros años como crítico Flores se orientó de manera definitiva a Europa y Estados Unidos, mostró una clara preferencia por autores nuevos y minoritarios y no prestó atención al desarrollo intelectual latinoamericano, algo que sí hizo a partir de finales de los años treinta cuando alcanzó progresivamente una mayor conciencia social, aumentó su implicación política y sus preferencias estéticas se decantaron hacia una literatura menos elitista y experimental, y más reivindicativa.

Flores llegó a Nueva York de su Puerto Rico natal con 18 o 19 años. En 1924 obtuvo un máster de Lafayette College con una tesis sobre José Enrique Rodó (el doctorado no lo recibiría hasta 1947 en Cornell University). En 1926 publicó su primer libro, Spanish Literature in English Translation: a Syllabus, un delgado manual donde, como indica su título, reunía y ordenaba la bibliografía existente en inglés sobre la literatura española, incluyendo traducciones. Pensando en sus tareas docentes, Flores exhibía aquí ya una preocupación por la existencia de textos hispánicos disponibles en inglés que no le abandonaría nunca y que cimentaría su fama como impulsor del hispanismo. En verano de 1928, y tras haber publicado una traducción de Los motivos de Proteo de Rodó (The Motives of Proteus, Brentano's, 1928) ${ }^{4}$, Flores visitó España y allí trabó amistad con Ramón

${ }^{4}$ En confidencia a Guillermo de Torre (carta del 9 de noviembre de 1930), Flores «disculpaba» haber hecho la traducción - lo que muestra su distanciamiento en esos mo- 
Gómez de la Serna ${ }^{5}$, a quien poco antes había dedicado un artículo en la revista literaria The Bookman, tras escribir sobre André Gide y Marcel Proust ${ }^{6}$. «At the Sign of Ramón», complementado con la traducción de un centenar de greguerías, presentaba a Ramón como un escritor vocacional, excesivo y fecundo que había alcanzado talla continental por su implacable capacidad para descubrir el alma de las cosas mediante el estilete del humor y una creatividad desbordante.

En 1929, Flores renunció a su puesto de instructor de español en Rutgers University para hacerse cargo de la dirección de la revista Alhambra, un proyecto de la efímera Hispano and American Alliance a fin de fomentar el interés en Nueva York por la cultura en español ${ }^{7}$. Pensada con periodicidad mensual, Alhambra publicó únicamente cuatro números entre junio de 1929 y enero de 1930, en que a decir del propio Flores (Carta a G. de Torre del 9 de noviembre de 1930) el crack del 29 dio al traste con ella, como meses antes le había pasado a la Revista de Estudios Hispánicos de Federico de Onís ${ }^{8}$. La mano de Flores puede detectarse a varios niveles. El efusivo editorial del primer número dejaba clara la voluntad de la revista de jugar a dos bandas y tender puentes, y mostraba la preferencia por la comunicación entre España y Estados Unidos, a pesar de que idealmente la Alliance buscaba fomentar el diálogo con todos los países de habla hispana: «Apretón de manos de dos razas... A los pueblos hispanos Alhambra aportará las mejores cosechas de las letras de los Estados Unidos e Inglaterra y, a la vez, traerá a los anglo-sajones los opíparos frutos de la Superespaña» ${ }^{9}$. La revista efectivamente combinó colaboraciones españolas y norteamericanas, y entre aquellas dio especial relevancia a los escritores jóvenes, ofreciendo las primeras versiones al inglés de Gerardo Diego, Ramón de Basterra, José Moreno Villa, Edgar Neville, K-Hito (seudónimo de Ricardo García López) o Federico García Lorca.

mentos de los ideales americanistas rodosianos - alegando que era un encargo irrenunciable en un momento de fuertes premuras económicas.

${ }^{5}$ Ramón le explicaría a Guillermo de Torre (por entonces en Buenos Aires) que Flores «va decidido a que sus Literaturas de vanguardia sean texto para los alumnos norteamericanos de su cátedra y ya ha pedido ejemplares a Mundo Latino» (Carta del 15 de julio de 1928, reproducida en García y Greco 2007: 133).

${ }^{6}$ Los artículos de Flores en The Bookman fueron: «André Gide and his "First Novel"», octubre de 1927, pp. 167-172; «Marcel Proust in Review», mayo de 1928, pp. 272-276; y «At the Sign of Ramón», junio de 1928, pp. 386-390.

7 Sobre la Alliance, vid. Anderson y Maurer (2013: 182-184).

${ }^{8}$ En 1991 Flores daba esta versión sobre el proyecto de la Alliance y la revista: «Yo dirigía en esa época una revista literaria que se llamaba Alhambra. Era una revista muy lujosa, bilingüe; el editor de la sección de arte era Gabriel García Maroto. La revista la patrocinaba un señor que resultó un pillo: no le pagaba a nadie. Este señor había querido reproducir el ambiente de la Alhambra en el piso onceavo de un edificio que se hallaba en la esquina de la calle 42 y la Quinta Avenida: importó mosaicos de España y mando construir una fuente. Quiso hacer una especie de Ateneo: había tertulias y ambiente español». (Flores 1991).

9 «Editorial», Alhambra, 1 (junio de 1929), p. 9. 
El tercer número de Alhambra incluyó dos romances de Lorca, seis fotografías y un artículo sobre él a cargo de Daniel Solana (seudónimo de G. García Maroto $)^{10}$. Flores había conocido al poeta granadino en su estancia neoyorquina a través de Federico de Onís y Ángel del Río. Mientras que las referencias de Lorca a Flores en su epistolario de esa época son escasas ${ }^{11}$, este último afirmó en una entrevista que tuvo «mucho trato» con el poeta, asiduo en su despacho de la Hispano and American Alliance donde había una tertulia semanal (Miguel Ángel Flores 1991). En otra entrevista Flores evocó al Lorca más seductor, aquel que cantando al piano conquistó a todos los invitados en una de las fiestas en su honor organizadas por Hershel Brickell (Talavera 1992: 13) ${ }^{12}$, por quien sabemos que a principios de octubre de 1929 Flores pensaba publicar la traducción de varios poemas de Lorca en la revista The Bookman ${ }^{13}$. Al parecer, Flores también fue la persona que llevó a Lorca a conocer al poeta Hart Crane ${ }^{14}$.

A principios de 1930, año en que ya trabajaba como profesor de español en la Universidad de Cornell, donde sustituyó a León Felipe, Flores publicó su segundo libro, Lope de Vega, Monster of Nature, una elaboración literaria de la vida del Fénix. La elección de un formato de biografía novelada mediante cuadros de época independientes y el uso de técnicas propias de la ficción provocó el elogio tanto en España como en Cuba, y el rechazo en Estados Unidos. Así, para $E l$ Sol era, por su amenidad y claridad, el libro que había faltado en el tricentenario ${ }^{15}$, y López Prudencio en $A B C$ resaltaba la vivaz recreación de época ${ }^{16}$. Lino Novás Calvo en Revista de Avance alabó su calidad poética, así como la selección de «los momentos más críticos» de una trayectoria vital ${ }^{17}$. Sirva como muestra de la recepción opuesta en Estados Unidos la opinión de Edward H. O'Neill, quien en su libro A History of American Biography 18001935 concluía: «there is too much fiction and too little criticism in the book to

10 Daniel Solana, «Federico García Lorca», Alhambra, 3 (agosto 1929), p. 24 y Lorca, «Ballads», p. 25.

${ }^{11}$ En cartas a su familia, Lorca cuenta que Gabriel García Maroto, León Felipe y Flores, «director de una revista editada en inglés de cosas españolas» (Anderson y Maurer 1997: 616), le llevaron una noche a descubrir Broadway, y también le nombra entre los que fueron a despedirle cuando partió hacia Vermont (Anderson y Maurer 1997: 639).

12 Brickell publicó sus recuerdos sobre Lorca en 1945: «A Spanish Poet en New York», $V Q R, 21.3$ (verano 1945), [s. p.], disponible en http://www.vqronline.org/essay/spanish-poetnew-york [ref. de 24/03/2016]. La perspectiva de Lorca sobre esas fiestas en Anderson y Maurer (1997: 629).

${ }_{13}$ Brickell se lo comenta al propio Lorca en carta del 7 de octubre de 1929, reproducida en Anderson y Maurer (2013: 69-70).

${ }^{14}$ Sobre este encuentro, véase Sahuquillo (2007: 79-80) y Anderson y Maurer (2013: 264-266). No hay ninguna referencia a él en el epistolario de Lorca.

15 T.B., «Bibliografía. Ángel Flores. La vida de Lope de Vega», El Sol. 6-VI-1936, p. 2.

16 José López Prudencio, «Lope de Vega», $A B C .6-\mathrm{V}-1935$, p. 16.

17 Lino Novás Calvo, «Lope de Vega, by Ángel Flores», Revista de Avance, 50, 15-XI1930, p. 285. 
make it of much value» $(1935: 298)^{18}$. Flores mostró enorme interés en que la obra llegara a España y merced a sus contactos con Guillermo de Torre finalmente la editorial «La Nave» la publicó en 1936 — en la resaca del tricentenario de la muerte de Lope - traducida por su valedor madrileño, quien algo más de una década después volvería a recuperarla para la editorial Losada. En el prólogo a la reedición de 1948, Guillermo de Torre (1948: 9) explicaba que el texto buscaba ofrecer una imagen de cuerpo entero de Lope a través de una técnica de estampas enlazadas por una leve trama novelesca.

1930 fue sin duda el año de Flores como traductor doble, pues vertió al español The Waste Land de Eliot y al inglés Cinelandia de Gómez de la Serna y Tres novelas ejemplares y un prólogo de Miguel de Unamuno. Respecto al primer libro, cabe señalar que un año antes Flores ya había presentado al público español a un escritor norteamericano, Sherwood Anderson, a través de un prólogo a la traducción de Poor White realizada por Julio Calvo Alfaro. Este breve texto contiene dos puntos importantes: una impugnación de la visión negativa de la literatura norteamericana que en realidad supone una invitación a practicar el nuevo internacionalismo de entreguerras. Frente al discurso antinorteamericano más usual - aireado incluso por algunos jóvenes vanguardistas - que se dedicaba a repetir «el consabido chiste acerca del canibalismo, la hipocresía, la insipidez yanqui» (Flores 1929: 2), Flores reclamaba una visión más abierta y más profunda de la realidad americana que explicaba a través del símil de un paseo por Nueva York. Caminando por sus calles se descubría el impulso juvenil y titánico de la modernidad en estado puro, la velocidad, el trasiego, la materialidad del esfuerzo y la urbanización de lo salvaje, y podía vislumbrarse la dirección del futuro, en lo que tenía a la par de seductor y de terrible, merced al imperio del dinero y de la técnica. Lo mismo ocurría a través de la lectura de la literatura norteamericana, que había superado su minoría de edad y ofrecía una nómina de escritores de primer rango en todos los géneros. Este cambio de percepción mediante la superación de prejuicios y tópicos era condición ineludible «si queremos llegar algún día a la realización de un cosmopolitismo intelectual y artístico» (Flores 1929: 2).

Pobre blanco había aparecido en la barcelonesa Editorial Cervantes y fue ahí donde, en forma de plaquette, se publicó Tierra baldía ${ }^{19}$. Flores trabajó en

${ }^{18}$ La reseña de Herschel Brickell para The New York Herald Tribune Books reconocía la originalidad y vivacidad en el tratamiento, pero criticaba su falta de aportes informativos y los momentos en que caía en anacronismos («The Prodigy of Old Spain», 20-IV-1930, p. 7). Del mismo tono fue la reseña en The New York Times («Lope de Vega, rival of Cervantes», 11-V-1930, p. 68) y aún más negativa la de The Brooklyn Daily Eagle («Lope de Vega, Up to Date», 30-VII-1930, p. 17).

${ }^{19}$ Luis Miguel Lázaro (2013) ha estudiado detalladamente el funcionamiento y el fondo de la editorial, señalando la edición de poesía como una de sus líneas distintivas, aunque sorprendentemente no hace ninguna mención al poema de Eliot. 
la traducción durante el año 1927. Según su propio testimonio, «lo hice como un ejercicio para saber qué tanto dominio tenía sobre el inglés» (Miguel Ángel Flores 1991) e incluso fue a Londres a conocer a Eliot ${ }^{20}$, quien le ayudó a desentrañar algunas cuestiones de interpretación a regañadientes, pues a su parecer «el poema debía leerse tal como lo escribió él» (Talavera 1992: 13), sin explicación ni divulgación. Eliot le dio el visto bueno a la traducción a finales de febrero de 1928 en una carta donde mostraba su preferencia porque el texto apareciera en Revista de Occidente, y de no ser posible, en La Gaceta Literaria $^{21}$.

La de Flores no era la primera traducción. El poema había aparecido en francés en mayo de 1926 en la revista Esprit, de la mano de Jean de Menasce, y en alemán en abril de 1927 en la Neue Schweizer Rundschau, en traducción de Ernst Robert Curtius, así como en holandés e italiano. Como ya indicó Howard Young, en la península ibérica la primera traducción fue al catalán, pues en 1927 apareció en L'Amic de les Arts una version sin firmar - pero muy probablemente a cargo de Marià Manent - de una parte del poema, la titulada «The Fire Sermon», aunque faltaban los versos 235 a 256 y además no se incluían las notas de Eliot $^{22}$. Flores tradujo el poema completo, así como todas las notas, y en un breve prólogo lo presentaba como el equivalente en verso del Ulises, la síntesis de una época donde quedaba «expuesto todo nuestro caos, toda nuestra angustia» (Flores 1930a: 7) de forma modernísima, aunando mitos de varias tradiciones, con técnica «cinematográfica» (8) y donde a menudo «las normas de expresión se rompen» (8) dando lugar a una proliferación de referencias culturales.

Ramón Gómez de la Serna saludó la aparición señalando «la dificultad pasmosa de revertir cada palabra al castellano» ${ }^{23}$ del texto original y El Sol señaló el «servicio meritísimo» que Flores le hacía a la literatura de ambos lados del Atlántico ${ }^{24}$. A decir de Howard Young el libro se vendió bien en Madrid, e incluso fue «chosen by a book club, one of whose judges was

${ }^{20}$ Las razones que Flores dio a Eliot para acometer la traducción fueron dos: una, que el texto era síntesis de la decadencia espiritual de toda una época; y dos, que «your poem promises much esthetically. The youth of the Spanish-speaking world is in search of new values, and I believe that The Wasteland in its Spanish avatar will be a welcomed gift from the English language» (Carta de Flores a Eliot del 30 de enero de 1928, citada fragmentariamente en Eliot, Letters 1928-1929, después de la carta de Eliot del 22 de febrero de 1928, [s.p.])

${ }^{21}$ Allí Eliot reconocía su conocimiento insuficiente del español para evaluar la traducción. En carta posterior a Erik Mesterton (16 de diciembre de 1931, Letters 1930-1931, p. 778), Eliot juzgaba buena la traducción española a pesar de contener algunos errores y reconocía no haber colaborado tan estrechamente con Flores como con Menasce y Curtius.

${ }^{22}$ Sobre la recepción de Eliot en Cataluña, véase Albert Manent (1997).

${ }^{23}$ Ramón Gómez de la Serna, «El año pombiano», La Gaceta Literaria, 97 (1931), pp. 10-11.

${ }^{24}$ «Eliot, Tierra baldia», El Sol. 14-I-1933, p. 2. 
Antonio Marichalar» (1994: 3) ${ }^{25}$. Numerosos críticos han señalado la impresión que en Lorca pudo causar el poema, así como «Los hombres huecos» traducido por León Felipe (y aparecido en Contemporáneos, núm. 33, 1931), lo que explicaría no solo el descubrimiento por parte de Lorca de la ciudad moderna como tema, sino que le pusiera Yerma al drama que estaba pensando. Por lo que respecta a Latinoamérica, José Emilio Pacheco considera la versión de Flores «el libro más influyente en la poesía mexicana del siglo veinte» (1992: 49).

Como es sabido, ese mismo año de 1930 apareció otra traducción al español de The Waste Land bajo el título «El páramo», completada por el escritor Enrique Munguía mientras trabajaba en el consulado de México en Ginebra. Munguía tomó varias decisiones cuando menos discutibles: vertió el poema original en prosa, argumentando que en español no existía equivalente prosódica del verso libre, y solo reprodujo once de las notas al pie de Eliot. Cuando el texto salió publicado en Contemporáneos (número 26-27, de julio-agosto, pp. 15-32), Munguía en una breve nota reconocía la preexistencia de la traducción de Flores, y a renglón seguido afirmaba contar con la autorización de Eliot para producir otra versión al español. Pero el asunto es un poco más complicado a la luz de la correspondencia de Eliot. Munguía le pidió autorización para traducir The Waste Land en una carta curiosamente sin datar a la que Eliot, que contestaba correspondencia con asiduidad, respondió el 8 de octubre de 1930 (Letters 1930-1931, p. 334) aludiendo a la preexistencia del texto de Flores y no obstante otorgando su permiso con una «condición necesaria»: poder revisar la traducción y hacer sugerencias antes de su publicación. Pero, a tenor de las fechas, «El páramo» ya había aparecido. El 20 de noviembre Flores escribió a Eliot molesto, pues creía que la versión de Munguía iba a dañar al propio Eliot, así como a la circulación de la edición española, a lo que el poeta respondió en carta del 9 de diciembre (Letters 1930-1931, p. 420) justificando la autorización por haberle sido Munguía presentado por un amigo común, para después lamentar, amagando con una protesta por escrito, que no se le hubiera permitido revisar la traducción.

Como traductor del español al inglés, Flores publicó en 1930 dos obras: Tres novelas ejemplares y un prólogo de Unamuno (Nueva York: Albert and Charles Boni), cuya traducción Arthur Livingston - agente de Blasco Ibáñez en Estados Unidos - venía negociando con distintas editoriales norteamericanas desde 192426. En el caso de la segunda, Movieland (Nueva York: The

${ }^{25}$ En relación al premio del «libro del mes», Young se basa en el testimonio del propio Flores, quien hablando con Miguel Ángel Flores añadió que Eliot recibió el dinero del premio. No he podido encontrar evidencia impresa al respecto. Por cierto que en 1934 Marichalar juzgaría la traducción de Eduardo Munguía «más prieta» (1934: 63) que la de Flores.

26 Vid. el artículo de Pereiro Otero y F. Plata (2012) sobre la relación entre Livingston y Unamuno, y el de Agramonte (1961) sobre las traducciones de Unamuno en Estados 
Macaulay Company), se trataba, como señalaba Ogier Preteceille en El Sol, de la primera versión en inglés de un libro de Ramón, ya traducido a casi una docena de lenguas ${ }^{27}$. Flores insertó una nota de presentación para el público americano. Ramón sobrepasaba «intellectual frontiers» (1930b: vii) gracias a la construcción de su propio personaje como literato y a un trabajo infatigable plasmado en una obra pasmosa. En ese sentido, debía incluírsele en una tradición española de personajes excesivos como Lope de Vega o Goya donde la pasión y el empuje eran los motores creativos principales frente a una razón en horas bajas. Pero en su dedicación exclusiva a la literatura, Ramón no sacralizaba esta, sino el desorden del mundo, que se proponía contar una y mil veces a base de disgregarlo en sus infinitos componentes y ponerlos a interaccionar en una danza de jovialidad. Flores destacaba además la faceta de conferenciante de Ramón y su invención de un nuevo género literario, «la greguería, a sort of metaphoric maxim or aphorism without any moralizing or academic heaviness» (1930b: xi), y concluía recordando que Movieland estaba escrita sin que su autor hubiera visitado nunca los Estados Unidos; precisamente por eso conseguía dar un certero retrato de Hollywood, pues no describía el de verdad, sino el imaginado por los espectadores ${ }^{28}$. Ese mismo año de 1930, Flores volvió a visitar España y por supuesto Pombo, como recordaba Ramón en su crónica del año pombiano 1930 para La Gaceta literaria, donde destacaba su labor como hispanista y traductor.

Por esas fechas, Flores empezó a pensar en el proyecto de escribir en inglés un resumen de literatura española contemporánea para la colección «One Hour», una iniciativa de la editorial Lippincott dirigida a ofrecer síntesis panorámicas sobre literatura, arte y cultura a cargo de reconocidos especialistas. Al parecer, se publicaron en total unos quince volúmenes, pero la colección parece haber cesado después de $1930^{29}$. Flores proyectaba el libro como superación

Unidos. Flores había publicado un fragmento en inglés de El espejo de la muerte en el último número de Alhambra (4, enero de 1930, pp. 5-6).

27 «Actualidad literaria en el extranjero. Estados Unidos», El Sol. 18-V-1930, p. 2. En Automoribundia, Ramón recordaba los pormenores de la publicación: «después de una larga correspondencia con Ángel Flores y de firmar un contrato de diez hojas con la casa Macaulay de Nueva York, sólo recibí 42 dólares, que como llegaron a mí cuando los bancos norteamericanos se fundían, me fui muy difícil poderlos cobrar» (cit. en García y Greco 2007: 133n).

${ }^{28}$ Lucien Zacharoff desde The Brooklyn Daily Eagle celebró la oportunidad para conocer a uno de los autores más reputados en Europa por su estilo y sus sorprendentes metáforas («Ibañez has a «succesor» in Sr. Ramon», 30-IV-1930, p. 23), algo en lo que coincidía The Boston Daily Globe («Spanish Authors writes of American Movieland», 26-IV-30, p. 13). Time también destacaba la fuerte personalidad de Ramón y tildaba el libro de «crude, violent, highly colored: a succession of journalistic pieces rather than a connected narrative» («Flame-Colored Spectacles», 7-IV-1930, p. 80).

29 Sobre literatura aparecieron An Hour of American Drama a cargo de Barrett H. Clark, An Hour of American Poetry de Charles Edward Russell, An Hour of the American Novel 
del que Jean Cassou había publicado en Francia (Panorama de la littérature espagnole contemporaine, París, Kra, 1929) y que no satisfizo a nadie ${ }^{30}$. Por carta a Guillermo de Torre (9 de noviembre de 1930) podemos saber algo más sobre las líneas maestras del volumen. Flores reconocía que la literatura española flojeaba en teatro y novela, por no existir autores comparables a Joyce, Gide o Proust, y pensaba centrarse en los siguientes nombres: Lorca, Alberti, Guillén y Salinas para la poesía; Baroja, Ramón, Chacel, Jarnés y Espina para la novela, y Jacinto Grau para el teatro. El proyecto nunca vio la luz así que es en los prólogos a antologías como Great Spanish Stories (1956) o Great Spanish Short Stories (1962) donde deben espigarse las opiniones generales de Flores sobre literatura española. Estas son usualmente esquemáticas por ubicarse donde se ubican, pero tienen la virtud - de la que muchas otras publicaciones de las mismas fechas carecen- de otorgar su lugar e importancia al esfuerzo de renovación cultural y estilística llevada a cabo por los integrantes de la joven literatura, poetas y prosistas, puros y engagées incluidos.

La labor infatigable de Flores también se plasmaba en la prensa periódica. Desde 1929 y por muchos años fue reseñador de literatura extranjera, fundamentalmente francesa y española, en The New York Herald Tribune Books. Ahí apartaba lo que llamaba las «petty national clasifications» ${ }^{31}$, consideraba la época de entreguerras «the most aesthetic period in history» ${ }^{32}$, marcada por la rápida sucesión de estilos, y en repetidas ocasiones defendía las virtudes de la novelística moderna que, liberada de «the well-known trappings and tricks of the old story-tellers $\rangle^{33}$, se alejaba de la pirotecnia adjetival, evitaba tópicos y frases gastadas y enfatizaba el rol activo del lector. Específicamente sobre literatura española, basta contraponer su elogio de la imaginación desbordante y la voluptuosidad lingüística à la Joyce de Tirano Banderas ${ }^{34}$ con su ataque al casticismo y aburguesamiento de la obra de Concha Espina ${ }^{35}$. Flores trabajaba para desmontar la imagen estereotípica de España, esa «same old uncharted Utopia discovered by romantic Frenchmen and American in the nineteenth century» ${ }^{36}$, todavía en boga a pesar de los esfuerzos de varias generaciones de escritores españoles. Por eso en su reseña de The European Caravan, Flores

de Grant M. Overton y una síntesis histórica sobre la novela inglesa a cargo de Ford Madox Ford, The English Novel: From the Earliest Days to the Death of Joseph Conrad.

${ }^{30}$ Flores había criticado duramente el libro en una reseña aparecida en Alhambra donde reprochaba a Cassou dedicar demasiadas páginas al siglo XIX y muy pocas a las jóvenes generaciones. «Littérature [E]Spagnole. Par Jean Cassou», Alhambra, 2 (Julio de 1929), pp. 82-83. Idéntica acusación, como veremos, le hizo al reseñar The European Caravan.

31 «Werfel's Best Work to Date», 20-XI-1932, p. 8.

32 «From a Frozen Heart», 19-I-1930, p. 2.

33 «A High-Speed Cagliostro», 29-XI-1931, p. 17.

34 «Valle-Inclán», 20-X-1929, p. 23.

35 «The Woman and the Sea», 5-VIII-1934, p. 7.

36 "“Romantic” Spain», The Saturday Review of Books, 14-VI-1930, p. 1127. 
consideraba la parte española la peor por la nefasta influencia de Jean Cassou, valedor de la ortodoxia y de los autores de mayor edad, lo que envejecía al volumen al no dar espacio suficiente a las «polyfacetical complexities» de la nueva literatura ${ }^{37}$.

Desde octubre de 1931 Flores contribuyó también a Books Abroad, la revista sobre literatura extranjera que la Universidad de Oklahoma había puesto en marcha en 1927. De sus textos destaca un elogioso artículo de 1932 sobre Benjamín Jarnés presentado como un autor cuya obra madura superaba la polémica entre arte deshumanizado y arte comprometido, pues ofrecía una visión a la par poética e irónica del presente a través de la mitopoesis: mitos que condensaban actitudes vitales de la época ${ }^{38}$.

A pesar de la delicada situación económica, en 1932 y con la ayuda de la Universidad de Cornell, Ángel Flores fundó The Dragon Press, una editorial en principio dedicada a escritores modernos minoritarios; allí vieron la luz The Knife of the Times \& other Stories de Williams Carlos Williams ${ }^{39}$ y la primera novela de Julian Shapiro, The Water Wheel. Pero el sello tuvo entre sus títulos el Diario de un seductor de Kierkegaard, una selección de rimas de Petrarca y The Anatomy of Don Quixote, una recopilación de estudios a cargo de Ramón Menéndez Pidal, A. Morel-Fatio, Helmut Hatzfel e Ivan Turgueniev, editada por el propio Flores junto a M. J. Benardete. En su introducción, Benardete situaba el libro en una doble tesitura: por una parte, una nueva ola de recuperación del Quijote por parte de académicos y estudiosos; por otra, una reivindicación de los valores contenidos en él —y que, en la línea de Ortega, sintetizaban la manera española de ver el mundo - como una posible guía frente a la desesperación del mundo contemporáneo: «Europe and America are exploring anew this realm of the spirit [...] because there is a realization, more or less dim, that Cervantes can aid us in our groping for a new hold of life and an insight into the unsolvable problems harrassing us» (Flores y Benardete 1932: xii-xiii). La aventura de The Dragon Press acabaría a principios de 1933 por razones pecuniarias, como William Carlos Williams le explicaba por carta a Louis Zukofsky: «even such a venture as Ángel Flores' Dragon press has cost its sponsor two or three thousand dollars which he'll never see again. It can't be done today» (carta del 24 de mayo de 1933, Ahearn 2003: 158). El interés de Flores por promocionar la joven literatura norteamericana se muestra también en el proyecto de una antología de cuentistas estadounidenses contemporáneos que propuso a Guillermo de Torre para Espasa-Calpe. Flores contactó

37 «The New Spirit in Literature», The New York Herald Tribune Books, 17-IV-1932, p. 9.

38 «Benjamín Jarnés», Books Abroad, 7.3 (Julio de 1933): 282-283.

39 En su autobiografía, Williams (1967: 298-299) recuerda que tras publicar el libro, Flores desapareció y nunca supo más de él, hasta que un amigo suyo le informó de que se estaban saldando los ejemplares. Curiosamente, Flores afirma en la entrevista con Talavera (1992: 14) que Williams «quedó encantado». 
con más de veinte escritores para que participaran en el proyecto y envió al crítico madrileño un cuento de Shapiro para la Revista de Occidente que nunca se publicó.

Flores no cejaba en su empeño de dar a conocer a los autores españoles en Estados Unidos, a pesar de que por esas fechas vivía un momento de reconfiguración de sus opiniones literarias, influenciadas por posicionamientos ideológicos que exigían un mayor compromiso político de los escritores. La correspondencia con Guillermo de Torre hace esto visible. En carta del 20 de octubre de 1932, Flores reiteraba su querencia por estilistas como Espina o Jarnés, cuyo humor ensalzaba, pero juzgaba artificiosos a Antonio de Oregón o Valentín Andrés Álvarez, por lo que prefería a alguien como Joaquín Arderíus a pesar de sus problemas de escritura. De hecho, Flores estaba negociando la publicación en Estados Unidos de Campesinos del mencionado Arderíus, La turbina de Arconada y Pero sin hijos de Salazar Chapela, ninguno de los cuales acabó apareciendo, y le solicitaba a su interlocutor español obras de Pilniak, Leonov o Plejanov. Es en ese año de 1932 que el crítico puertorriqueño acarició la idea de un volumen antológico titulado Contemporary Spanish Authors. Del proyecto sabemos que dejaba fuera a autores ya conocidos en inglés, como Pérez de Ayala, Gabriel Miró o Ramón Gómez de la Serna, que debía incluir un prólogo del autor de Literaturas europeas de vanguardia, y que Flores solicitó directamente a varios autores jóvenes como R. J. Sender, F. Ayala, César M. Arconada o E. Salazar Chapela que espigaran sus páginas mejores. Al parecer estos no eran especialmente receptivos y tardaban en enviar sus selecciones, o como en el caso de Salazar Chapela nunca lo hicieron. No ayudó que ese mismo año apareciera Great Spanish Short Stories, Representing the Work of the Leading Spanish Writers of the Day (Boston, Houghton Mifflin) ${ }^{40}$, una antología de casi 400 páginas editada por Warre Bradley Wells ${ }^{41}$ con prólogo de Henri Barbusse y notas biográficas de Julián Gómez García bajo su seudónimo de J. Gorkin. Flores acusó al libro de ofrecer una imagen falseada de la literatura española, pues no mostraba adecuadamente su evolución en las últimas décadas, y además se escoraba hacia el radicalismo político (los pocos textos ajenos a éste, a cargo de Miró, Jarnés y Espina, eran para el reseñista los mejores), convirtiendo el volumen en «insipid propaganda $»^{42}$. El proyecto de antología seguía vivo a principios de 1933 con el título Contemporary Spanish Fiction. Flores se mostraba contento con los textos de Jarnés y Obregón (unas páginas de Efectos navales) y menos con

${ }^{40}$ El libro fue publicado simultáneamente en Londres por Eyre \& Spottiswoode bajo el título The Spanish Omnibus.

${ }^{41}$ Wells vertió al inglés originales en francés y en español, entre estos últimos, obras de Azorín, Eugeni d'Ors, Huidobro y Marañón, y en 1934 publicó un libro sobre el rey Alfonso XIII.

42 «Union Square's Spain», The New York Herald Tribune Books, 3-IV-1932, p. 11. 
«El baño de la muerta» de Arderíus y la selección de Siete domingos rojos de Sender ${ }^{43}$, que prefería cambiar por un fragmento de Imán. A mediados de 1933 la antología estaba terminada, con la excepción del capítulo de Salazar Chapela, una nota biográfica sobre Díaz Fernández, y el prólogo. Como ya se adelantó, el libro nunca salió y años después Flores seguramente recicló buena parte del material para otras empresas antológicas como Heart of Europe (1943) o Spanish Writers in Exile (1947).

En el año 1933, incómodo ante la situación en los Estados Unidos y en precaria situación laboral ${ }^{44}$, Flores acarició la idea de trasladarse a España y vivir de las traducciones, los artículos o la docencia, pero aconsejado por de Torre desistió. Ese mismo año apareció Authors Today and Yesterday, una enciclopedia de escritores contemporáneos en la que Flores se encargó de varias entradas sobre autores franceses (Giradoux, Romains), italianos (Svevo) y también españoles: Ortega y Gasset — en cuyo retrato defiende a la Revista de Occidente de las acusaciones de ser «snobbish and Un-Spanish» (Kunitz 1933: 508), anotando que había descubierto a alguno de los escritores jóvenes más importantes de España-, Ramón Pérez de Ayala — clásico a la par que castizo-, Azorín — uno de los mayores escritores impresionistas europeos- o Ramón Gómez de la Serna —el escritor vocacional por excelencia, sempiterno humorista— ${ }^{45}$. Por esas fechas, Flores tiene casi terminado un libro sobre la evolución de la novela contemporánea en Europa entre 1930 y 1934. Además, gestiona la publicación en la revista Europe de un extracto del trabajo de Guillermo de Torre Itinerario de la nueva pintura española y completa una antología de textos de Benjamín Jarnés (carta a de Torre del 25 de junio de 1933), libro que tampoco se publicó, además de por las dificultades editoriales, porque tanto Jarnés como Ortega y Gasset hicieron caso omiso a la petición de un prólogo. Un ejemplo más de lo que Flores entendía como falta de interés de los escritores españoles por su difusión en los Estados Unidos, y que le hacía flaquear en lo que él mismo denominaba su «propaganda pro-España» (carta a de Torre del 8 de enero de 1934).

A la búsqueda de un nuevo canal de diseminación para la literatura europea contemporánea, Flores puso en marcha en mayo de 1934 junto a críticos como Samuel Putnam, Stanley Kunitz o Louis Kronenberger la revista The Literary World, según de Torre en Luz «un periódico mensual consagrado a las letras

${ }^{43}$ A pesar de ello, en febrero de 1935 el periódico La Libertad informaba de que Flores estaba preparando la traducción inglesa de La noche de las cien cabezas, que nunca apareció. («Dos libros de Sender al inglés», 3-II-1935, p. 7.)

${ }^{4}$ A principios de 1933, Federico de Onís informaba por carta a León Felipe de que «Flores me ha escrito diciéndonos que el año que viene perderá su puesto en Cornell» (texto reproducido en Anderson y Maurer 2013: 194).

${ }^{45}$ Los artículos de Flores sobre literatura española en este volumen son los dedicados a Azorín (pp. 33-38), Ramón Gómez de la Serna (pp. 268-272), José Ortega y Gasset (pp. 506-509), Ramón Pérez de Ayala (pp. 516-519) y Valle-Inclán (pp. 658-661). 
modernas de Europa, bellamente presentado ${ }^{46}$. En sus nueve números Flores incluyó a Ortega y Gasset (con un «mensaje a la juventud»), Jarnés (del que hizo la presentación y tradujo un fragmento autobiográfico) y Antonio de Obregón (del que se publicó un cuento, «Folletín»). La revista destacó por dar a conocer a Franz Kafka en Estados Unidos a los diez años de su muerte a través de un monográfico (número 3, julio de 1934) en el que colaboraron, entre otros, Thomas Mann, Denis Saurat, Conrad Aiken, Max Brod y Waldo Frank. Flores había conocido a Kafka a través de la traducción de La metamorfosis aparecida en Revista de Occidente (números 18 y 19 de 1925) y se convertiría en uno de sus escritores de referencia, al que dedicó en años posteriores varios estudios y recopilatorios bibliográficos.

Los temas presentes en The Literary World (el escritor y la guerra, la literatura burguesa, el compromiso del intelectual) así como el peso de la literatura germana y rusa muestran un perfil mucho más politizado de Flores que se iría acentuando. Dentro de la órbita del Partido Comunista, la labor de Flores en esos años se plasmó en tres frentes principales: uno, las clases y conferencias para obreros, así por ejemplo los cursos sobre «tendencias sociales en la literatura contemporánea» y «teorías literarias» impartidos en 1937 en la New York Worker's School. Dos, la organización en Nueva York de The Critics Group, una reunión de críticos y profesores dedicados a publicar estudios de interpretación marxista de autores clásicos como Shakespeare, Balzac, Ibsen, Gogol o Cervantes. Los libros, a decir del propio Flores, se vendían muy bien, y por eso «creíamos que la revolución iba a llegar de un momento a otro» (Talavera 1992: 14). Y, en tercer lugar, sus colaboraciones en revistas de izquierda como New Masses (1935) Art Front (1936, y de la que era parte del comité editorial) y la propia revista de The Critics Group, Dialectics (1937-1939).

En paralelo a esta implicación política se produjo una doble evolución en el crítico: por una parte, sus preferencias estéticas tendían a obras más conectadas con la realidad y que plasmaban los conflictos sociales, con personajes menos cerebrales y más activos y donde disminuía la dificultad formal y la elaboración estilística. Si en 1928 Flores había llamado a la tríada formada por La tierra baldia, Ulises y En busca del tiempo perdido «the Sacred Books of our century» ${ }^{47}$, seis años después se burlaba de los críticos que «once tracked Mr. Eliot's wasteland allusions to their original sources and later went into ecstasy over Mr. Joyce's philological calembours» y elogiaba la «readability», «clearness» e «interesting narrative» de Los hombres de buena voluntad, el ciclo narrativo de Jules Romains ${ }^{48}$. Por otra parte, la atención a los conflictos políticos, a las nuevas formas de autoritarismo y a las tensiones geopolíticas

\footnotetext{
46 «Noticiario breve», Luz. 23-V-1934, p. 7.

47 «Marcel Proust in Review», The Bookman, mayo de 1928, pp. 276.

48 «Jules Romains, Materialist and Mystic», The New York Herald Tribune Books, 1410-1934, p. 6.
} 
provocó la ampliación de la mirada hacia la realidad social de los países latinoamericanos, donde las masas además de la dominación del sistema capitalista soportaban la opresión del imperialismo. Un proyecto frustrado de revista que Flores impulsó a mediados de 1935 y que debía titularse The Americas tenía como propósito fundamental dar voz a las reivindicaciones de los países del Caribe y Sudamérica.

A mediados de los treinta apareció la última colaboración de Flores en publicaciones españolas, una panorámica sobre la vida editorial norteamericana en 1934 para el Almanaque Literario para 1935 de Plutarco. Su texto, de estilo cortante y frases contundentes, muestra en general escepticismo cuando no franco pesimismo, y presenta un paisaje caracterizado por la continuación de la crisis de ventas del libro, la poca calidad de las nuevas obras de autores consagrados como Waldo Frank o Sinclair Lewis, el éxito de los libros de historia, economía y política, así como de la novela proletaria y revolucionaria, y el impacto prácticamente nulo de las traducciones de autores españoles.

En 1936 se publicó finalmente en español la biografía de Lope de Vega y el periódico El Sol retrató a Flores como «ensayista y crítico de firme personalidad, muy favorablemente conocido ya en nuestros medios intelectuales por su labor de hispanista, en la que descuellan sus traducciones al inglés de Ramón Gómez de la Serna y de algunos otros escritores jóvenes» ${ }^{49}$. Semanas después de iniciada la guerra civil española Flores mostró su claro posicionamiento en un artículo sobre el teatro republicano ${ }^{50}$ y poco tiempo después la editorial de The Critics Group publicó doce poemas de Rafael Alberti bajo el título $A$ Spectre is Haunting Europe, los beneficios de cuya venta iban destinados a las tropas antifranquistas. En un breve prólogo Flores presenta a Alberti como el más importante poeta revolucionario de España, capaz de combinar virtuosismo formal, compromiso y honda conexión con el proletariado, o como resume en una frase que nuevamente revela la evolución del propio Flores, «a T. S. Eliot or a Hart Crane who had read with understanding The Communist Manifesto» ${ }^{51}$ (Flores 1936b: 10). Todavía en 1938 adaptó Peribañez y el comendador de Ocaña para la escena americana, pero no existe constancia de que llegara a representarse. En esas fechas Flores pasaba por una situación de desempleo y de depresión que concluyó cuando soslayando sus simpatías comunistas lo llamaron para colaborar con la Division of Intellectual Cooperation de la Pan American Union, una institución pública con sede en Washington D.C. dedicada a estrechar los lazos culturales entre los Estados Unidos y los países latinoamericanos y que había tomado nuevos bríos desde la «good neighbour

\footnotetext{
49 T.B., «Bibliografía. Ángel Flores. La vida de Lope de Vega», El Sol. 6-VI-1936, p. 2.

50 Ángel Flores y Ben Ossa, «Spain, 1936. The Theatre in Arms!», New Theatre (septiembre 1936): 1.

${ }^{51}$ Como hemos visto, las alusiones negativas a Eliot, de quien tan cerca había estado, parecen servir a Flores para exorcizar sus preferencias estéticas de unos pocos años atrás.
} 
policy» puesta en marcha por el presidente Franklin D. Roosevelt en 1933. Flores se encargó de diversas tareas de traducción, gestión de originales y coordinación de intercambios bibliográficos de dos de las revistas de la institución, Points of View y Panorama: A record of Inter-American Events. A partir de ese momento, aunque sin abandonar sus intereses en la literatura europea, Flores centró cada vez más sus actividades en la difusión de la literatura de los países americanos de habla hispana. Todavía en 1944 publicó una traducción de «El río fiel» de Benjamín Jarnés para el Quarterly Review of Literature $^{52}$ y un año antes había colaborado en la ambiciosa antología The Heart of Europe. An Anthology of Creative Writing in Europe 1920-1940, editada por Klaus Mann y Hermann Kesten. Esta elegía a toda una época de la literatura del viejo continente, publicada en el nuevo, incluía un capítulo sobre España con textos breves que iban de Unamuno y Machado a Jorge Guillén y Rafael Alberti. En una sucinta introducción, Flores señalaba cómo la producción peninsular había ido progresivamente sincronizándose con la de sus pares europeos, en un proceso de transformación de la lengua literaria dramáticamente truncado por la guerra civil. En privado y ante el enfado de Guillermo de Torre por la selección de autores — coja en prosistas de la joven literatura, tratándose de un volumen sobre la producción de entreguerras-, Flores confesó las precarias condiciones de producción del volumen:

Dicha obra se hizo durante la guerra a toda prisa. Ya se había ido Klaus para la guerra y Kesten andaba de prisa por terminar la faena: insistió en Unamuno y Ortega para los ensayistas y lo demás poesía y ficción, a condición de que todo estuviese ya traducido. Así es que se escogió lo ya preparado que entresaqué de revistas o de mis archivos, y a fin de cuentas usaron solo la mitad de lo que les di ${ }^{53}$.

En 1942 había aparecido de la mano de Flores y de Dudley Poore Fiesta in November (Houghton), la primera antología en inglés de narrativa latinoamericana, a la que siguieron traducciones de Miguel A. Menéndez, Germán Arciniegas o Pablo Neruda. Flores, reincorporado ya a la enseñanza universitaria (era profesor en Queens College desde 1945), concentraba cada vez más sus esfuerzos en promocionar a los escritores de América Latina, aunque todavía tendría tiempo en 1947 de publicar la antología Spanish Writers in Exile (Benn Porter). Un año después Flores ensayaba una suerte de resumen de su trayectoria: «su contribución más fecunda ha sido en el campo de la traducción, dando a conocer en versión inglesa las obras más diversas del genio hispánico (y cita desde Lope a Jarnés). A la vez ha dado a conocer en lengua española La tierra baldía de T.S. Eliot y a numerosos poetas norteamericanos» (carta a de Torre del 30 de abril de 1948). Dos años después le confesaría al mismo

\footnotetext{
52 «The Faithful River», Quarterly Review of Literature, 2.1 (1944): 22-35.

53 Carta de Ángel Flores a Guillermo de Torre del 22 de diciembre de 1946.
} 
destinatario: «mi especialización es tan restringida que no permite darme el lujo de escribir sobre autores españoles» (carta de junio de 1950). Se iniciaba así una nueva etapa en la vida de Ángel Flores - espoleada por su famosa conferencia de 1954 sobre «El realismo mágico en la narrativa hispanoamericana»que iba a durar hasta sus últimos días y donde su implicación con la literatura europea en general y española en particular se haría desde un punto de vista docente e historizante, con la publicación de ediciones y antologías para el público universitario, volcando sus esfuerzos por difundir los desarrollos pasados y presentes de la literatura producida en Latinoamérica, a partir de la organización de congresos y encuentros de autores y profesores. Una labor que le convertiría en uno de los más reconocidos expertos en literatura latinoamericana de Estados Unidos.

\section{FUENTES}

Eliot, T. S. (2013). Letters, volume 4, 1928-1929. New Haven: Yale University Press.

Eliot, T. S. (2014). Letters, volume 5, 1930-1931. Londres: Faber and Faber.

Flores, Ángel (1926). Spanish Literature in English Translation. A Bibliographical Syllabus. Nueva York: H. W. Wilson.

Flores, Ángel (1929). «Prólogo», en Sherwood Anderson, Pobre blanco. Barcelona: Editorial Cervantes, pp. 1-4.

Flores, Ángel (1930a). «Prólogo», en T.S. Eliot, La tierra baldía. Barcelona: Editorial Cervantes, pp. 7-9.

Flores, Ángel (1930b). «Translator's note», en Ramón Gómez de la Serna, Movieland. Nueva York: The Macaulay Company, pp. vii-xii.

Flores, Ángel (1930c). Lope de Vega, Monster of Nature. Nueva York: Brentano's.

Flores, Ángel y M. J. Benardete (1932). The Anatomy of Don Quixote: A symposium. Nueva York: Dragon Press.

Flores, Ángel (1934). «Norteamérica», en Miguel Pérez Ferrero, Esteban Salazar Chapela y Guillermo de Torre (eds), Almanaque literario 1935. Madrid: Plutarco, pp. 240-243.

Flores, Ángel (1936a). Lope. Madrid: Ediciones La Nave.

Flores, Ángel (1936b). «Introduction», en Rafael Alberti, A Spectre is Haunting Europe. Poems of Revolutionary Spain. Nueva York: Critics Group, pp. 9-11.

Flores, Ángel y Dudley Poore (eds.) (1942). Fiesta in November. Stories from Latin Ameri$c a$. Boston: Houghton Mifflin.

Flores, Ángel (ed.) (1948). Spanish Writers in Exile. Berkeley: Ben Porter.

Flores, Ángel (ed.) (1955). Great Spanish Short Stories. Nueva York: Modern Library.

Mann, Klaus y Hermann Kesten (eds.) (1943). Heart of Europe. An Anthology of Creative Writing in Europe 1920-1940. Nueva York: L. B. Fischer.

\section{BIBLIOGRAFÍA CITADA}

Agramonte, Roberto (1961). «Unamuno en Norteamérica», La Torre: Revista General de la Universidad de Puerto Rico. 9, pp. 563-582. 
Ahearn, Barry (ed.) (2003). The Correspondence of William Carlos Williams and Louis Zukofsky. Middletown, CT: Wesleyan UP.

Anderson, Andrew A. y Christopher Maurer (eds.) (1997). Federico García Lorca. Epistolario completo. Madrid: Cátedra.

Anderson, Andrew A. y Christopher Maurer (eds.) (2013). Federico García Lorca en Nueva York y La Habana: Cartas y recuerdos. Barcelona: Galaxia Gutemberg/Círculo de Lectores.

Cornejo, Josefina (2012). «Ángel Flores», El Trujamán. Revista diaria de traducción. 9 de febrero. Accesible en: $<\mathrm{http} / / / \mathrm{cvc}$.cervantes.es/trujaman/anteriores/febrero_12/09022012. $\mathrm{htm}>$ [ref. de 25/03/2016].

Flores, Miguel Ángel (1991). «Ángel Flores, traductor de Lorca, Eliot, Neruda, puente entre

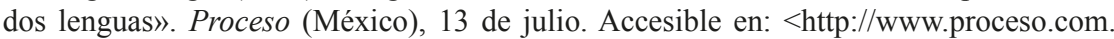
$\mathrm{mx} / 157474 /$ Ángel-florestraductor-de-lorca-ellot-neruda-puente-entre-dos-lenguas $>$ [ref. de 16/03/2016].

García, Carlos y Martín Greco (eds.) (2007). Escribidores y náufragos. Correspondencia Ramón Gómez de la Serna/Guillermo de Torre 1916-1963. Madrid - Frankfurt am Main: Iberoamericana - Vervuert.

Herrero-Senés, Juan (2015). «Samuel Putnam, cosmopolitismo y vanguardia española en Estados Unidos», Hispanic Review. 83 (4), pp. 403-421.

Kunitz, Stanley J. (ed.) (1933). Authors Today and Yesterday. Nueva York: The H.W. Wilson Company.

Lázaro, Luis Miguel (2013). «L'edició popular a Espanya. El cas de l'Editorial Cervantes. Notes», Educació i Història: Revista d'Història de l'Educació. 22, pp. 33-63.

Manent, Albert Manent (1997). «La recepció de l'obra de T. S. Eliot a Catalunya» en Del noucentisme a l'exili: sobre la cultura catalana del nou-cents. Barcelona: Publicacions de l'Abadia de Montserrat, pp. 227-240.

Marichalar, Antonio (1934). «Lancelot Andrews», Cruz y Raya. 12, pp. 61-64.

O’Neill, Edward H. (1935) A History of American Biography 1800-1935. Filadelfia: University of Pennsylvania Press.

Pacheco, José Emilio (1992). «Ángel Flores en el puente de Brooklyn». Proceso (México). 823, pp. 48-49.

Pereiro Otero, J.M. y Francisco Plata (2012). «Práctica y teoría de la traducción en Unamuno: Cuatro Cartas Inéditas», Anales de literatura española contemporánea. XXXVII, 3, pp. 1059-1115.

Sahuquillo, Ángel (2007). Federico Garcia Lorca and the Culture of Male Homosexuality. Jefferson, NC y Londres: McFarland \& Company.

Talavera, Laura (1992). «De Puerto Rico a Nueva York. Entrevista con Ángel Flores», Cultura Sur. 34, pp. 12-14.

Torre, Guillermo de (1948). «Prólogo» a Ángel Flores, Vida de Lope de Vega. Buenos Aires: Ed. Losada, pp. 7-18.

Williams, William Carlos (1967). Autobiography. Nueva York: New Directions.

Young, Howard (1994). «Introduction», en K. M. Sibbald y Howard Young (eds.), T.S. Eliot and Hispanic Modernity. Boulder: Society of Spanish and Spanish-American Studies, pp. 1-8.

Fecha de recepción: 5 de mayo de 2016

Fecha de aceptación: 22 de febrero de 2017.

Revista de Literatura, 2019, vol. LXXXI, n. ${ }^{\circ}$ 161, 207-225, ISSN: 0034-849X

https://doi.org/10.3989/revliteratura.2019.01.009 